\title{
Rhabdomyolysis and acute kidney injury secondary to rosuvastatin use
}

\author{
Miriam Hassani* \\ Broomfield Hospital, CT1 Anaesthetics, United Kingdom
}

\begin{abstract}
A 66 year old man with a previous episode of acute kidney injury (AKI) secondary to NSAID use that had resolved entirely, was admitted with AKI, proximal myopathy and oliguria. His creatine kinase (CK) was almost 12,000 on admission, creatinine 513 and an ultrasound of his kidneys, ureters and bladder (US KUB) showed no urinary tract obstruction. He was dialysed three times and his Rosuvastatin was omitted from his regular medications from day 2 of his admission. None of his other regular medications were known to have any nephrotoxic or muscular effects. After extensive investigation and improvement in his condition following omission of the statin, he was diagnosed with Rosuvastatin induced rhabdomyolysis and AKI. On follow-up the patient's symptoms had resolved and his renal function back to baseline. This case report highlights the danger of Rosuvastatin induced rhabdomyolysis and its presentation in patients.
\end{abstract}

\section{Background}

Statins are associated with muscle complaints ranging from muscle weakness and cramps to myalgia with and without elevated creatine kinase (CK) levels, mild CK elevations, or myositis and rhabdomyolysis [1]. Myalgia is the least severe but most common presentation of muscle toxicity. Rhabdomyolysis and potential renal failure are the most severe but least common presentations. In general, the most efficacious statins are well tolerated by the majority of patients, even at maximal doses, and serious myopathy and rhabdomyolysis with statin treatment are rare. Statin-associated myopathy is generally a concern when patients have more than one risk factor for muscle syndromes, such as an elderly patient with poor renal function. Caution should be used when starting statin therapy in patients with more than one risk factor (e.g., elderly with poor renal function). Patients should be informed about the risk of myopathy and advised to report any unexplained muscle discomfort or weakness that is unassociated with physical exercise or other trauma to muscles [2].

\section{Case presentation}

A 66 year old man was admitted on a general medical take, following referral by his GP with a ten day history of pain and difficulty walking upstairs, weakness in his thighs, bilateral leg swelling and lethargy. He also reported reduced urine output over the last four days with no dysuria or haematuria and denied any fever or abdominal pain. Bloods taken by the GP the day prior to admission revealed a creatinine of 425 .

Eight months earlier, he had been admitted with AKI secondary to one week of NSAID use. This episode resolved by stopping the NSAIDs and his renal function returned back to his baseline of around 100. His past medical history was of colon cancer in 2009 (resected), open angle glaucoma, diverticulitis and duodenal and colonic polyps. He had no significant family history of note.

On this admission the patient denied any recent use of NSAIDS, over-the-counter medication or recent antibiotic use. He complained of increasing fatigue and reduced appetite over the last week or so but stated he was still drinking more than a litre of water a day. On examination he had bilateral pitting oedema to his knees but nil else of note and his observations were within normal range. His bloods taken on admission to the Emergency Assessment Unit (EAU) showed his creatinine was 513 and his CK was 12,000.

The patient's regular medications were as follows:

Lansoprazole $30 \mathrm{~g}$ daily

Co-Codamol 30/500 when required

Tramadol $50 \mathrm{mg}$ when required

Rosuvastatin $20 \mathrm{mg}$ at night

Lumigan eye drops

Metoclopramide $10 \mathrm{mg}$ when required

\section{Treatment}

The patient was catheterised and his fluid input/output strictly recorded. The following day the patient's care was taken over by the renal team, who stopped his Rosuvastatin. A series of investigations were ordered including immunology studies and urine protein:creatinine ratio and his CK monitored daily. Ultrasound Kidney/Ureter/Bladder was normal. The patient was put on fluid restriction of $1 \mathrm{~L}$ a day and his urine output in the last 24 hours was just $130 \mathrm{mls}$.

The patient was dialysed for 2 hours the day following admission $(10 / 4 / 15)$ due to worsening renal function and oliguria. After the first session of dialysis, creatinine levels had come down to 480 and the CK was 9,900 . The patient was dialysed again twice more on $11 / 4 / 15$ and $13 / 4 / 15$. He was started on oral sodium bicarbonate $500 \mathrm{mg}$ three times

Correspondence to: Miriam Hassani, Broomfield Hospital, CT1 Anaesthetics, 2 Chestnuts, Hutton, Brentwood, Essex, CM13 2PA, UK, Tel: 07947687298, E-mail: Miriam.hassani89@gmail.com

Received: January 23, 2016; Accepted: February 18, 2016; Published: February 22, 2016 
a day and his weight measured daily. On admission his weight was 111 $\mathrm{kg}$ (usual weight $102 \mathrm{~kg}$ ).

Over the next week following the dialysis sessions, the patient's renal function began to recover, with the creatinine falling each day. The peripheral oedema improved with the help of the Frusemide and mobilisation and daily weights showed a decrease down to his premorbid weight. He was seen by the physiotherapists daily and at the point of discharge he was able to walk with the help of a walking stick. His creatinine had come down to 217 and he was discharged home. The diagnosis was made as rhabdomyolysis and AKI secondary to Rosuvastatin.

The patient was seen in follow-up clinic three weeks later and was well in himself with no ongoing symptoms. His creatinine was back down to 97 and he had no pitting oedema, therefore the Frusemide was stopped. The proximal myopathy had greatly improved although he still had some ongoing weakness but was able to walk independently again.

\section{Discussion}

In 2009 Khan and Ibrahim reported a case of Rosuvastatin induced rhabdomyolysis and AKI in a low risk patient after he presented with a five day history of generalised muscle weakness and easy fatigability. His creatinine was 140 and creatine kinase (CK) of 4566 U/L. The Rosuvastatin was stopped on the first day of admission and the patient started on intravenous fluids with careful monitoring of serum electrolytes. In this case the patient did not require dialysis and the patient's renal function began to improve the day after admission without statins and on follow-up his creatinine was back to his baseline and the patient was on diet therapy rather than re-started on statins [3].

In 2012 a case was reported of Rosuvastatin induced rhabdomyolysis in a 62 year old man who presented with a one week history of bilateral thigh, back and shoulder pain, easy fatigability and passing dark urine. His creatinine was 240 and CK 21,210 U/L. As with the previous case, the patient was low risk, the Rosuvastatin stopped on the day of admission and intravenous fluids started. The creatinine returned towards normal the day after admission and the patient did not require dialysis. On follow-up the patient had no ongoing symptoms and his renal function had completely resolved. Statins were not re-started and instead the hypercholesterolaemia was managed with diet therapy [4].

\section{Learning points}

- Clinicians should maintain an increased level of awareness of the possibility for muscle toxicity and rhabdomyolysis, even in low doses and with low risk patients.

- Patients who take Rosuvastatin and present with muscle pain or weakness should have their renal function tested as well as their CK levels to investigate for rhabdomyolysis

- Those patients who present with similar symptoms as the patient in this report (proximal myopathy, lethergy, reduced urine output) and are taking Rosuvastatin should have this medication stopped immediately pending further investigation into their symptoms

\section{References}

1. Sathasivam S, Lecky B (2008) Statin induced myopathy. BMJ 337: a2286.[Crossref]

2. Talbert RL (2006) Safety issues with statin therapy. J Am Pharm Assoc (2003) 46: 479-488.[Crossref]

3. Khan FY, Ibrahim W (2009) Rosuvastatin induced rhabdomyolysis in a low risk patient: a case report and review of the literature. CurrClinPharmacol 4: 1-3.[Crossref]

4. Jasotharan V, Pirasath S, Sundaresan KT (2012) Rosuvastatin induced rhabdomyolysisRare case reported in Batticaloa. International Journal of Medicine and Medical Sciences 2: 269-270.

Copyright: (C2016 Hassani M. This is an open-access article distributed under the terms of the Creative Commons Attribution License, which permits unrestricted use, distribution, and reproduction in any medium, provided the original author and source are credited. 